\title{
QoS Improvement for the Next Generation Heterogeneous Network
}

\author{
G. Vijayalakshmy \\ Perunthalaivar Kamarajar \\ Institute of Engineering and \\ Technology Pondicherry \\ University India
}

\author{
N. Lakshmy \\ Pondicherry Engineering \\ College Pondicherry University \\ India
}

\author{
G. Sivaradje \\ Pondicherry Engineering \\ College Pondicherry University \\ India
}

\begin{abstract}
Next Generation Wireless Networks (NGWNs) focus on convergence of different Radio Access Technologies (RATs) providing good Quality of Service (QoS) for applications such as Voice over IP (VoIP) and video streaming. A heterogeneous network is to enable the users to obtain and share necessary and timely information in the right form over integrated heterogeneous network which is scalable and evolvable. Load balancing which is a significant method to achieve the resource sharing and IPQoS algorithm in the heterogeneous network is used to improve the overall performance of the network by configuring of queuing methods. Existing interworking networks couldn't support the bandwidth demands of many multimedia applications which exceed the capacity of the interworking network. To meet the challenges, LTE is a step toward the 4th generation of radio technologies designed to increase the capacity and speed of mobile telephone networks. The LTE promises to be one of the wireless access technologies capable of supporting very high bandwidth applications. In this paper a hybrid coupled interworking of three networks (WiMAX - WLAN -LTE IPQoS -LB) using H.323 signaling protocol is proposed. Heterogeneous network model based on Fast handover Hierarchical Mobile IPV6 (FHMIPV6) protocol that integrates the WiMAX, LTE and WLAN technologies is proposed to improve QoS. The QoS parameter in terms multimedia application such as traffic sent and received, RTP, response time, jitter, packet end -to-end delay, TCP delay, Ethernet delay, packet delay variation, of proposed work were simulated and its performance are measured.
\end{abstract}

\section{Keywords}

LTE, WiMAX, WLAN, Hybrid Coupled, FHMIP.

\section{INTRODUCTION}

The evolution towards the Next Generation Wireless Networks (NGWNs) has led to an interesting paradigm shift where the user is no longer passive but could influence the selection of the wireless access network. In the last few decades, various wireless and mobile networks have been developed and deployed across the world [1, 2]. These networks have different access technologies and have been designed to work independently without cooperating with each other. Seamless interworking between these wireless networks, either directly or over a common IP based backbone is an ultimate objective of the upcoming Heterogeneous Wireless Access Network (HWAN), also known as NGWN [3]. Wireless access techniques are continuously expanding their transmission bandwidth, coverage, and Quality of Service (QoS) support in recent years. One of the major challenge to (HWAN) and 4G wireless network is to support QoS [4] due to the different channel characteristics, various access controls, varying bit rate, bandwidth allocation methods, fault tolerant levels and handoff methods, protocols and supports. QoS support can occur at access level, packet level, transaction level, circuit level, core network and connectivity level as well as user level [5]. In a complete wireless solution, the End-to-End communication between two or multiple users will likely involve multiple wireless networks, with different types of accessing techniques, as well as the underlying IP-based networks [6]. These different characteristics of radio access technologies can complement each other through interworking between heterogeneous networks.

The necessity for uninterrupted communication when the mobile device moves from one location to another one calls for a new technology. This kind of communication can be effectively implemented using Mobile IP [7]. The advantage of mobile IP is its physical layer independence, which means that any communication media, including wired and wireless networks will support mobile IP [6]. Mobile IP will provide major benefits, including application transparency and the possibility of seamless roaming. Hybrid coupled interworking is very much useful in the case of resource sharing and traffic management and thereby enhances the QoS performance [7]. In this paper a novel hybrid coupled interworking model that integrates Worldwide Microwave Access network (WiMAX), a Wireless Local Area Network (WLAN) and a Long Term Evolution network (LTE) is proposed with IPQoS and LB [8]. Heterogeneous network model based on Fast handover Hierarchical Mobile IPV6 (FHMIPV6) [9] protocol that integrates the WiMAX, LTE and WLAN technologies is proposed to improve QoS. The QoS parameter in terms multimedia application such as traffic sent and received, RTP, response time, jitter, packet end -to-end delay, packet delay variation, is simulated and its performance were measured.

The rest of the paper is organized as follows. Section II presents an overview of load balancing, while Section III overviews about Hybrid coupled interworking of WiMAX WLAN networks with LB and IPQoS. In Section IV proposed hybrid coupled WiMAX - WLAN-LTE interworking architecture with LB and IPQoS is presented. Section V presents the proposed hybrid coupled WiMAX-WLAN-LTELB-IPQoS-FHMIP. Simulation results that are used to evaluate the performance of proposed architecture are presented in Section VI. Finally the paper is concluded with conclusion.

\section{LOAD BALANCING AND IPQOS 2.1 Load Balancing}

Load Balancing (LB) can act as load distributer for Internet Protocol (IP) Multimedia traffic across multiple servers [10]. In a load balancing scheme two or more servers can be incorporated. The requests are forwarded to another server when it gets overloaded. LB allows multiple servers to handle multiple requests simultaneously. The service time is reduced by using a LB to identify the server for the appropriate 
availability to receive the traffic. Distribution of service requests across a cluster of server can also provide by LB [11]. It also provides a cost-effective, efficient, and transparent method to expand the bandwidth of network devices and servers, to increase the throughput, and enhance data process capability, increasing the flexibility and availability of networks. Figure 1 shows the signal flow diagram of load balancer [10].

\section{A. Signal flow of Load Balancer}

Step 1: The user connects to the Internet and requests a service as shown in figure 1

Step 2: DNS routes the user to a specific IP address at a specific datacenter

Step 3: The user is connected to the load balancer.

Step 4: The connection is accepted by the LB and after deciding which server should receive the connection, it also

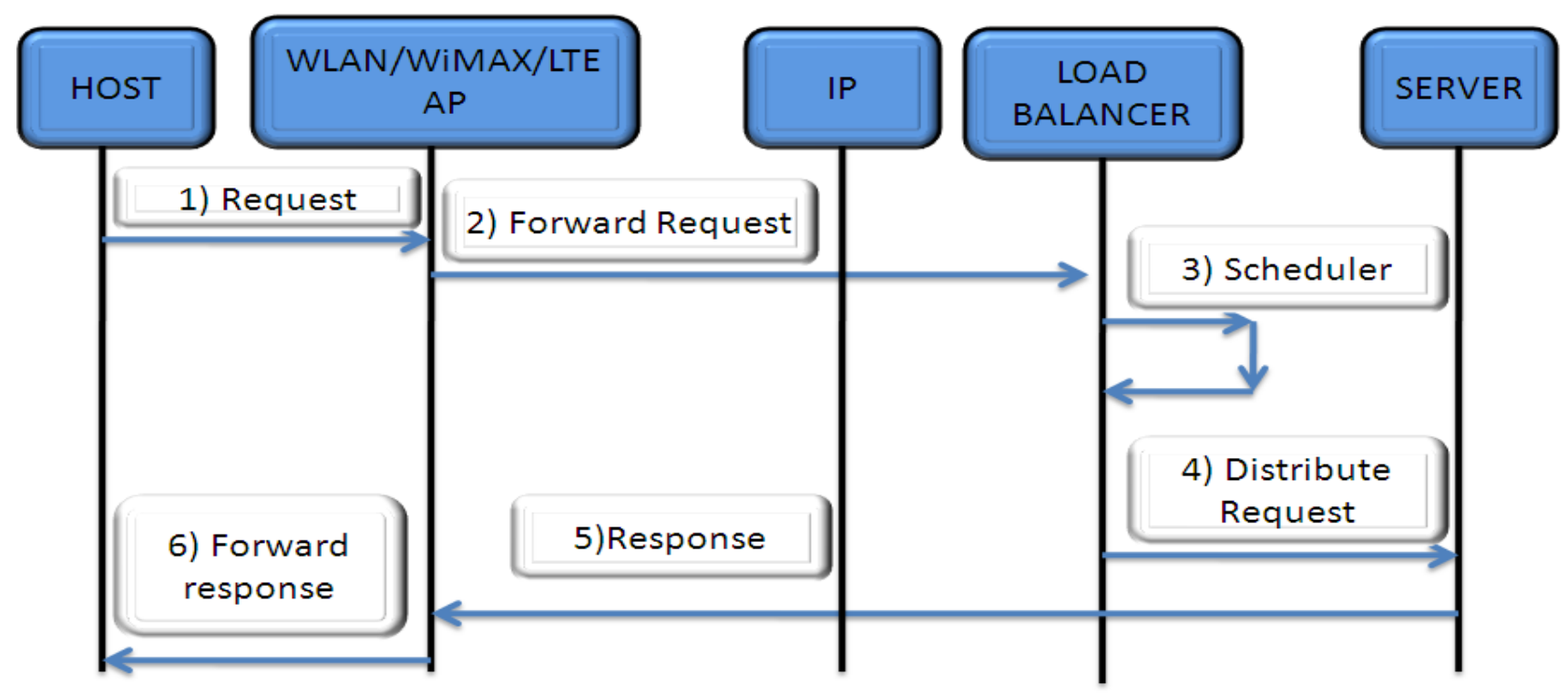

Figure 1: Signal flow of load balancer changes the destination IP to match the service of the selected host.

Step 5: The connection is accepted by the server and responds back to the original source, the client, via its default route, the LB.

Step 6: The return packet from the host is accepted by LB and it forwards the packet back to the client after changing the source IP address to match the virtual server IP and its port.

Step 7: After receiving the return packet, client check for identity from virtual server and displays the content. 


\section{HYBRID COUPLED INTERWORKING OF WIMAX - WLAN NETWORKS WITH \\ LB AND IPQOS}

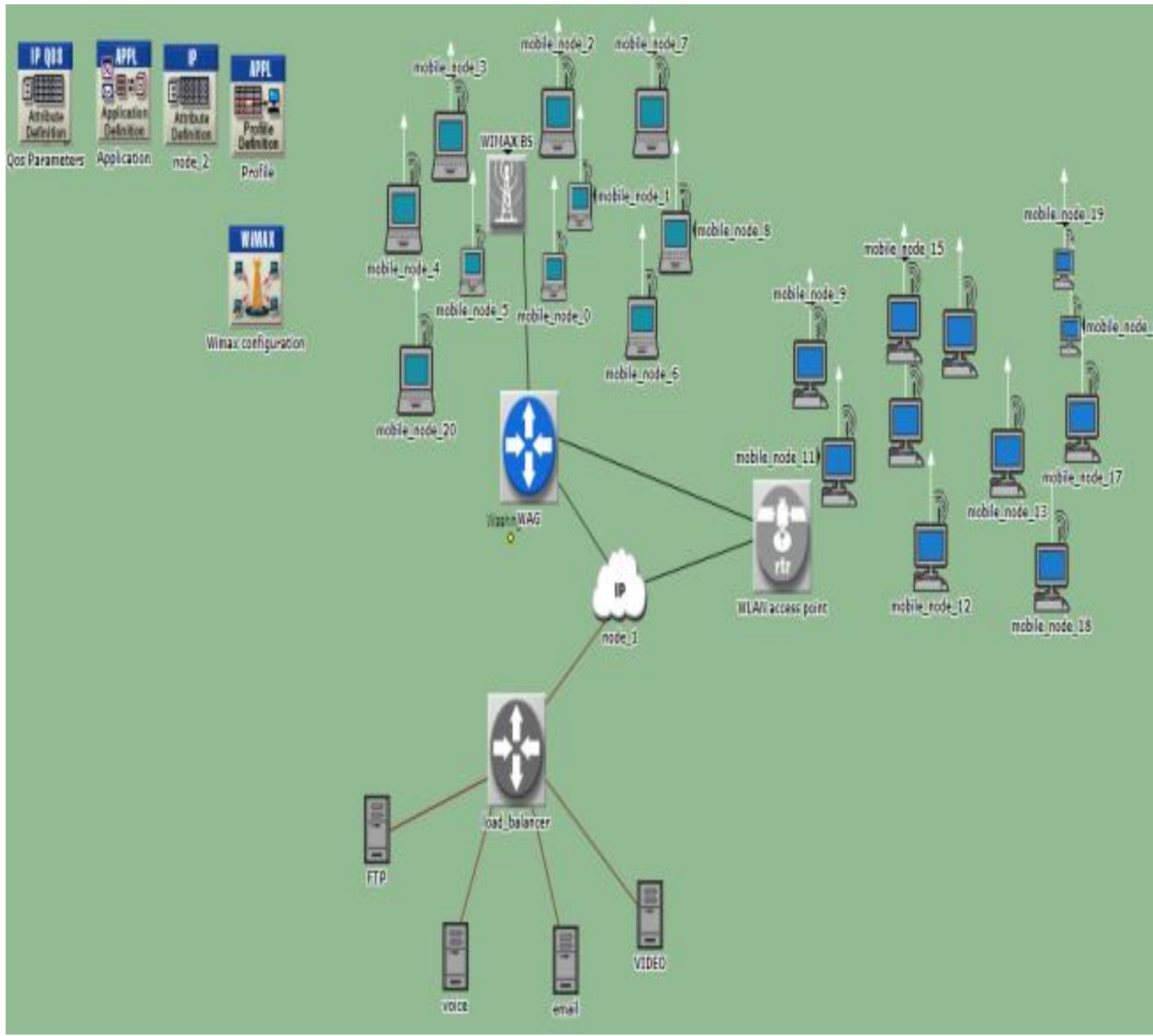

Figure 2: Hybrid coupled interworking of WiMAX- WLAN -LB-IPQOS

Hybrid coupling is the combination of the tight and loose coupled architecture. It has the advantage of tight and loose coupled networks [12, 13]. Resource sharing is take place dependently and independently according to the traffic condition. Hybrid coupled interworking is very much useful in the case of resource sharing and traffic management and thereby enhances the QoS performance. Figure 2 shows the Hybrid Coupled interworking of WiMAX-WLAN-LB-IPQOS were four servers are connected to the internet through hub. There are 20 users in the WiMAX and WLAN base station and access point. By means of hybrid coupling they can share the resources when the traffic congestion occurs. WiMAX has defined many interworking strategies between WiMAX and others wireless systems. After the user requested application is connected to coverage area of the integrated network by internet applications. Those applications have to be very much fast enough to reach the user from the server. Without considering Load balancing, there is the problem of delay in access and leads to decrease in QoS performance. Due to decrease in QoS performance, moving to load balancing in order to get speed of accessing the data from the server. Load balancer is used to share the load across server and thereby increasing the accessing speed of the integrated network. The LB has to share the traffic congestion at peak hour of the user needs. Number of connections coming from the integrated network has to be engaged by the load balancer. The LB not only balances the load across the server but also balance the network traffic. 


\section{PROPOSED HYBRID COUPLED INTERWORKING OF WLAN-WIMAX- LTE LB-IPQOS}

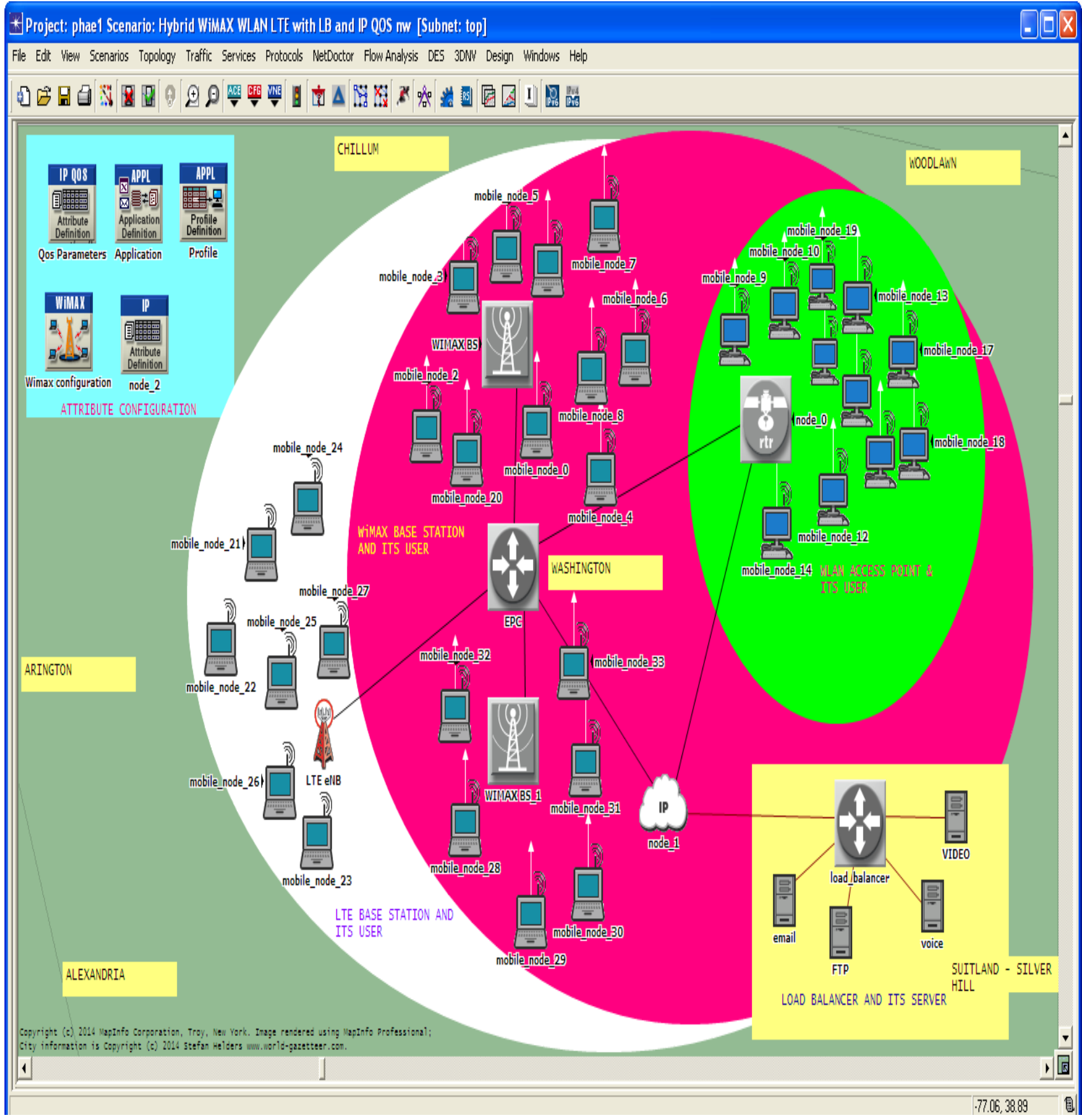

Figure 3: Hybrid Coupled Interworking of WLAN-WiMAX-LTE-LB-IPQoS

Figure 3 shows the proposed Hybrid coupled interworking of three networks WLAN-WiMAX- LTE with load balancing and IPQOS. Hybrid coupled the combination of hybrid coupled and loosely coupled networks [14]. The main advantage is that the network uses both direct and indirect path. The gateway which is used to integrate three networks by means of EPC and it can act as a main gateway to carry 


\section{PROPOSED FAST HANDOVER HIERARCHICAL MOBILE IPV6 (FHMIPV6)}

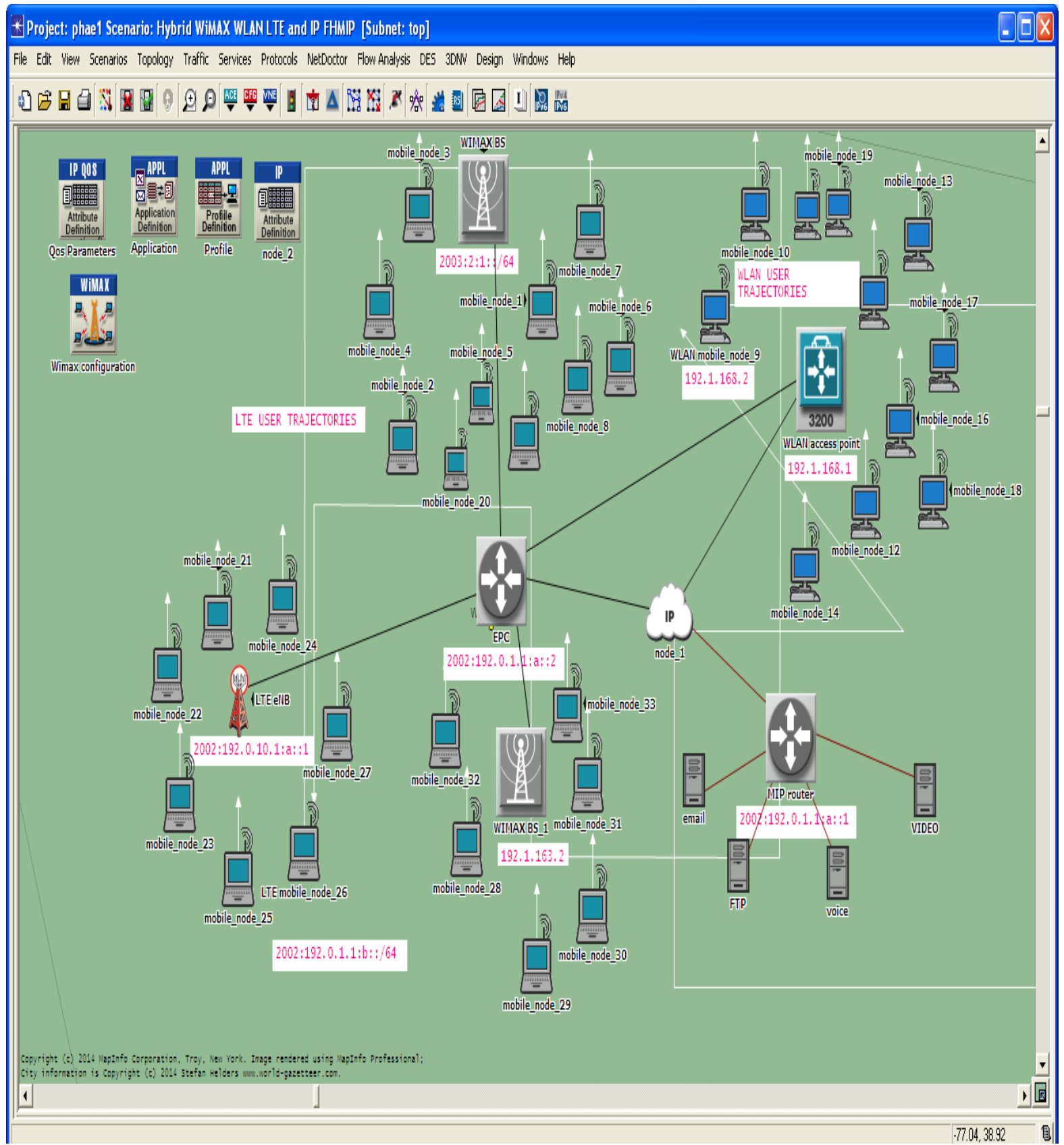

Figure 4: Hybrid Coupled Interworking of WLAN-WiMAX-LTE-LB-IPQoS -FHM IPv6

Figure 4 show the hybrid coupled interworking of three networks with FHMIPv6 and it has assigned IP address for each individual access point and its user. For example MIP router in the above scenario is allotted the following IP address 2002:192:0:1: a::1 The Internet Engineering Task Force (IETF) has proposed Mobile IPv6 (MIPv6) as the main protocol for mobility management at the IP layer. However, MIPv6 has some well-known drawbacks such as signaling traffic overhead, especially when the home agent (HA) or the correspondent node $(\mathrm{CN})$ is located geographically far away from the mobile node $(\mathrm{MN})$. Message transmission time for binding update registration will become very high resulting in long delay (handover latency) and high packet loss rate thereby causing user-perceptible deterioration of real-time traffic. Several extensions such as Fast Handovers for MIPv6 (FMIPv6) [9] and Hierarchical MIPv6 (HMIPv6), have been proposed to enhance MIPv6 performance. Combination of HMIPv6 and FMIPv6 motivates the design of Fast Handover for Hierarchical Mobile IPv6 (F-HMIPv6) protocol [17]. It combines between more efficient network bandwidth usage of 
HMIPv6 and the less handover latency and packet loss of FMIPv6. Figure 3 shows the network model of F-HMIPv6 along with the integration of three networks. A domain is managed by a network entity called Mobility Anchor Point (MAP). While entering a MAP domain, the MN receives router advertisements containing information about local MAPs from Access Routers (ARs) within range. Then, the MN obtains two care of addresses (CoAs): an on link Local CoA (LCoA) and a Regional CoA (RCoA) within the selected MAP domain. Then, a Local Binding Update (LBU) message is sent to the MAP to bind the MN's LCoA with its RCoA. Upon receipt of a successful Binding Acknowledgment (BA), the MN updates the binding of its RCoA with the HA and each $\mathrm{CN}$ [7]. As a result, packets destined to the $\mathrm{MN}$ are intercepted by the MAP, encapsulated and forwarded to the MN's on-link address. A movement within the MAP domain merely incurs LBUs at the MAP without further propagation to the $\mathrm{HA}$ and every $\mathrm{CN}$, thus significantly reducing the signaling load and micro-mobility related handover delays.
FMIPv6 is designed to enable the MN to rapidly detect its movements and to obtain a prospective IP address with a new AR while being connected to a current AR [9].

\section{PERFORMANCE EVALUATION}

The QoS parameter in terms multimedia application such as traffic sent and received, RTP, response time, jitter, packet end -to-end delay, TCP delay, Ethernet delay, packet delay variation, of proposed work were simulated and its performance are measured using OPNET.

\subsection{Results and Discussion}

Here in the performance analysis, $\mathrm{X}$ axis is taken as simulation time period and $\mathrm{Y}$ axis is taken as corresponding Parameter. Video conferencing traffic received is the average traffic arrival rate to all Video Conferencing applications deployed in the network. The statistic is computed based on the application data.

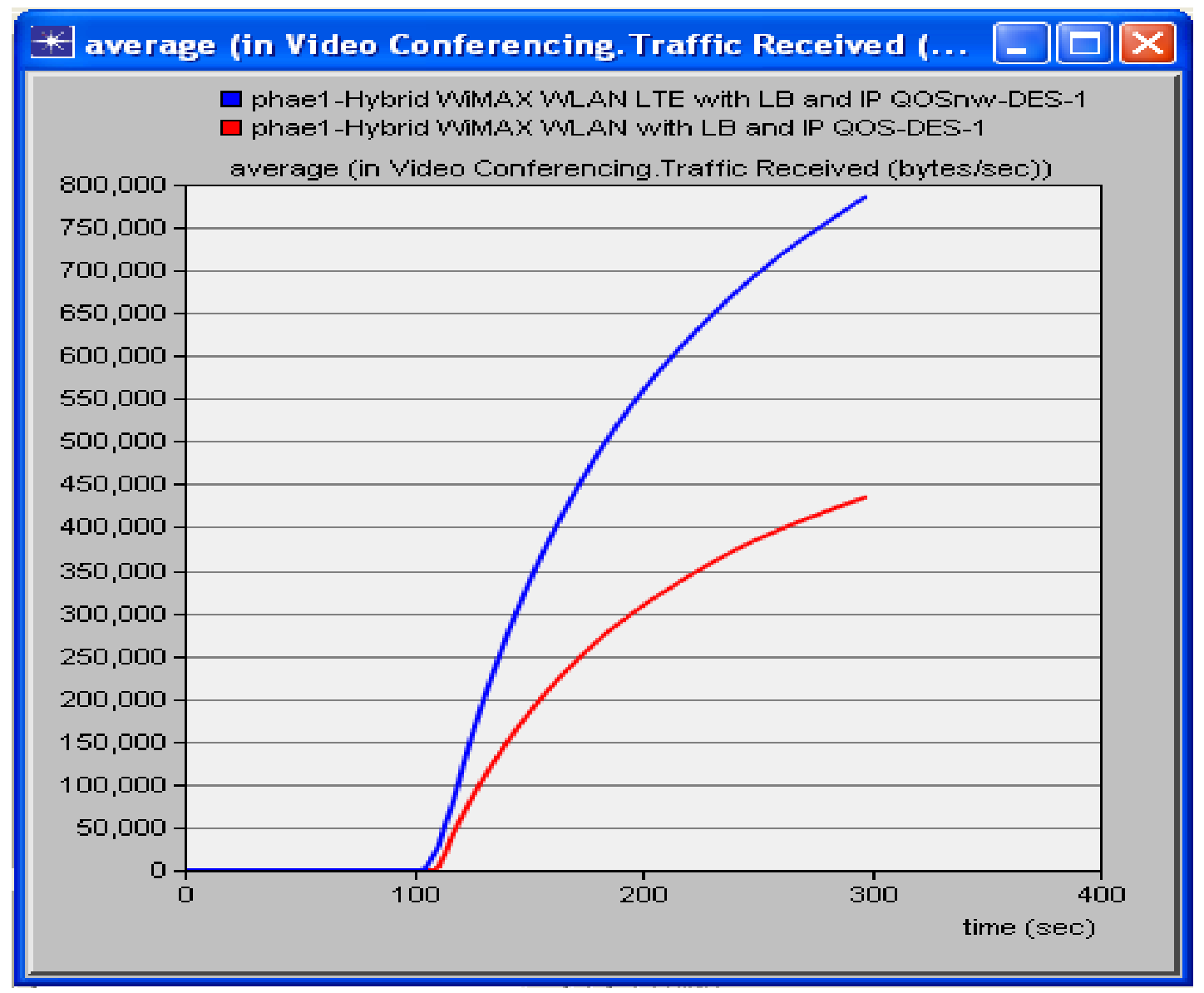

Figure 5: Video Conferencing Traffic Received (bytes/sec) for Hybrid coupled WiMAX-WLAN-LTE-LB-IPQOS and Hybrid coupled WiMAX-WLAN-LB-IPQOS

Figure shows 5 the Video Conferencing Traffic received in hybrid coupled WiMAX-WLAN-LTE-LB-IPQoS and hybrid coupled WiMAX-WLAN-LB-IPQoS. It is observed that hybrid coupled WiMAX-WLAN-LTE-LB-IPQoS carries 1500000 bytes/sec traffic reception compared to hybrid coupled WiMAX-WLAN-LB-IPQoS of 600000 bytes/sec traffic. Voice Jitter is the variation in the time between packets arriving, caused by network congestion, timing drift, or route changes. 


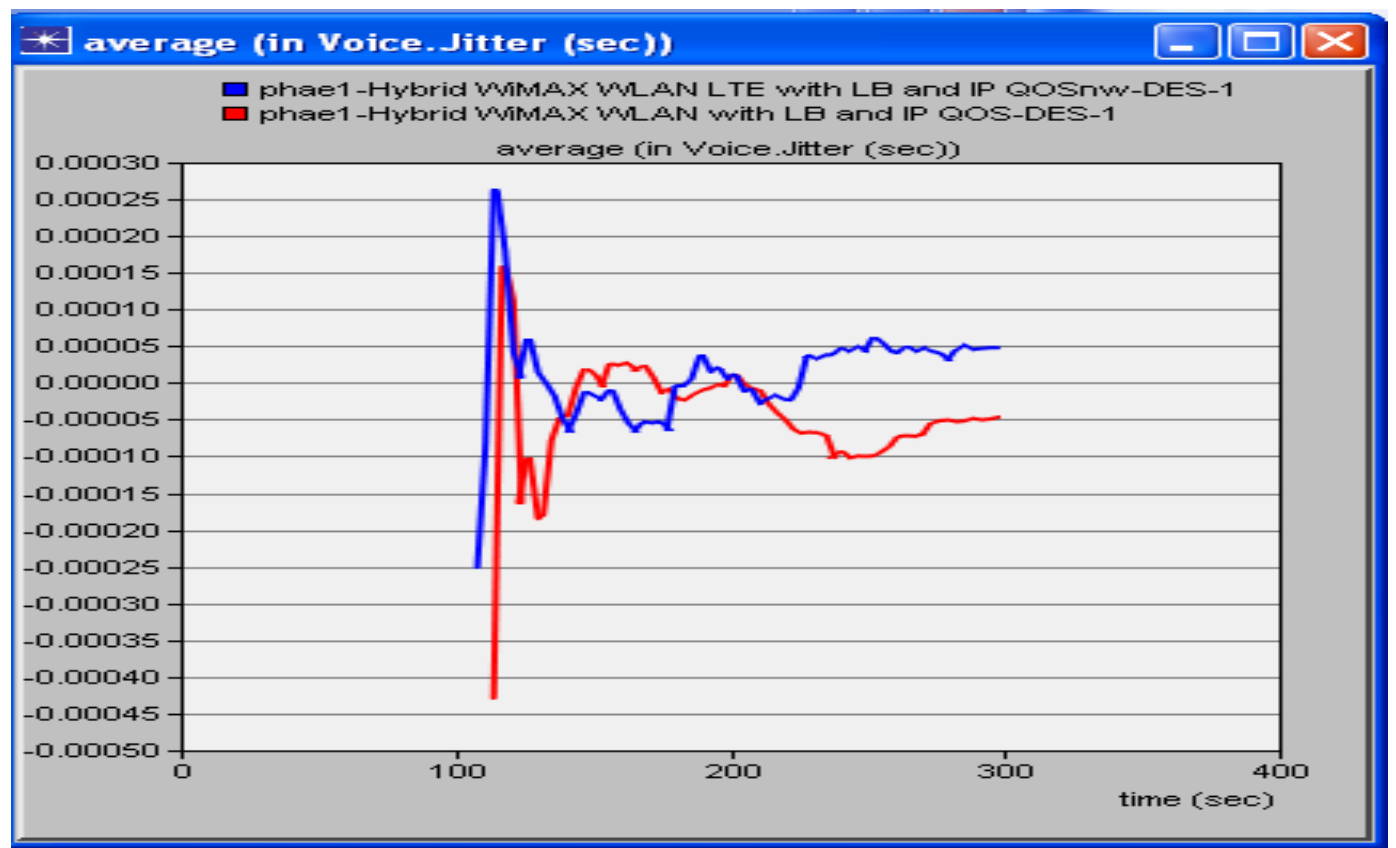

Figure 6: Voice jitter (sec) for Hybrid coupled WiMAX-WLAN-LTE-LB-IPQOS and Hybrid coupled WiMAX-WLAN-LBIPQOS

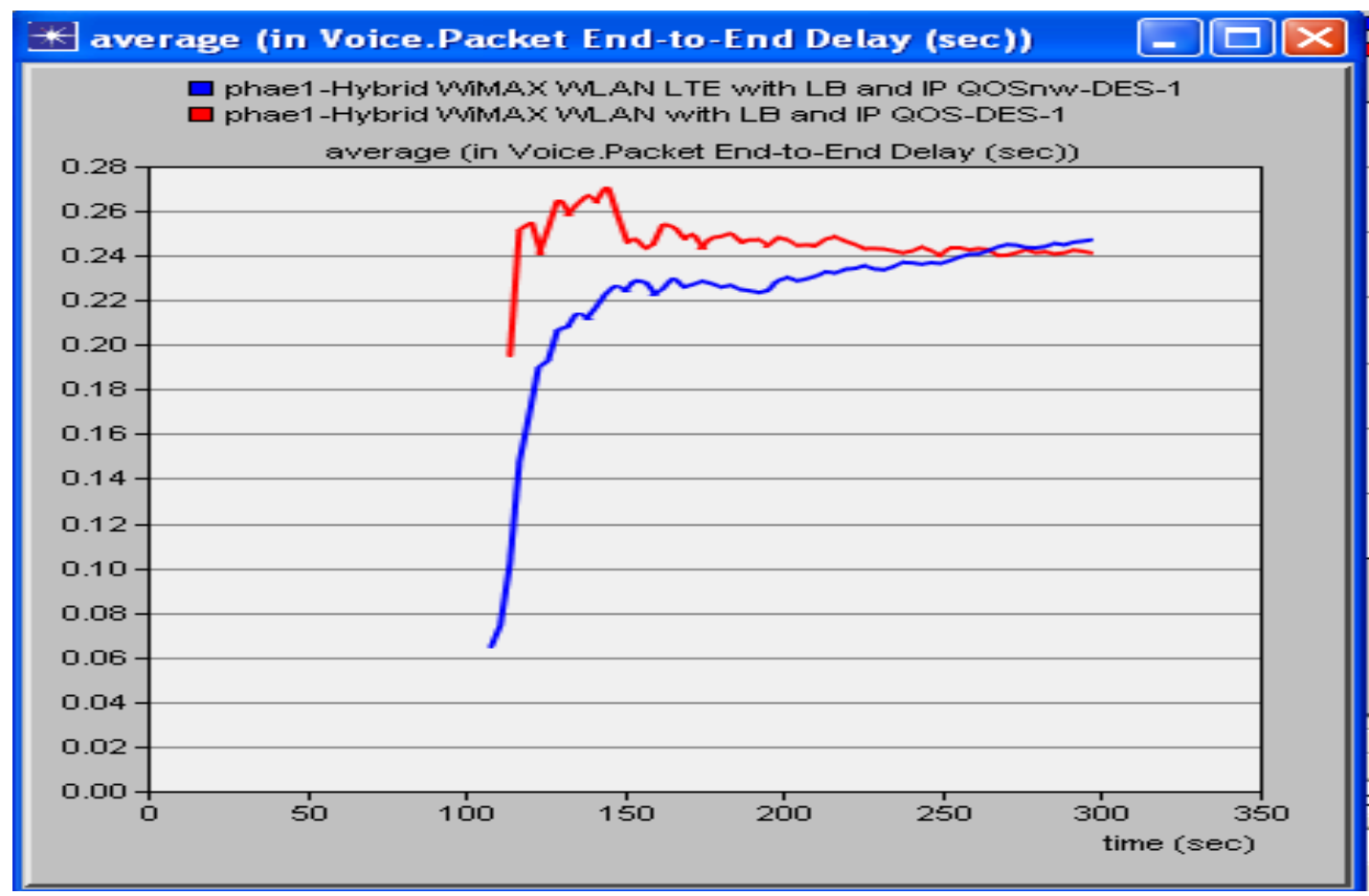

Figure 7: Voice Packet End to End delay (sec) for Hybrid coupled WiMAX-WLAN-LTE-LB-IPQOS and Hybrid coupled WiMAX-WLAN-LB-IPQOS

Figure 6 shows the Voice jitter in hybrid coupled WiMAXWLAN-LTE-LB-IPQoS and hybrid coupled WiMAXWLAN-LB-IPQoS. It is observed that hybrid coupled WiMAX-WLAN-LTE-LB-IPQoS has high jitter compared to hybrid coupled WiMAX-WLAN-LB-IPQoS. The variation in the jitter value is inacceptable limit. Voice Packet End-to-End Delay total is the delay experienced by the voice packets. It includes network, encoding/decoding, and compression delays. This statistic records data collected from all the nodes in the network. Figure 7 shows the Voice Packet End-to-End Delay in hybrid coupled WiMAX-WLAN-LTE-LB-IPQoS and hybrid coupled WiMAX-WLAN-LB-IPQoS. It is observed that hybrid coupled WiMAX-WLAN-LTE-LBIPQoS has less delay of $0.23 \mathrm{sec}$ compared to hybrid coupled WiMAX-WLAN-LB-IPQoS which has $0.27 \mathrm{sec}$. 


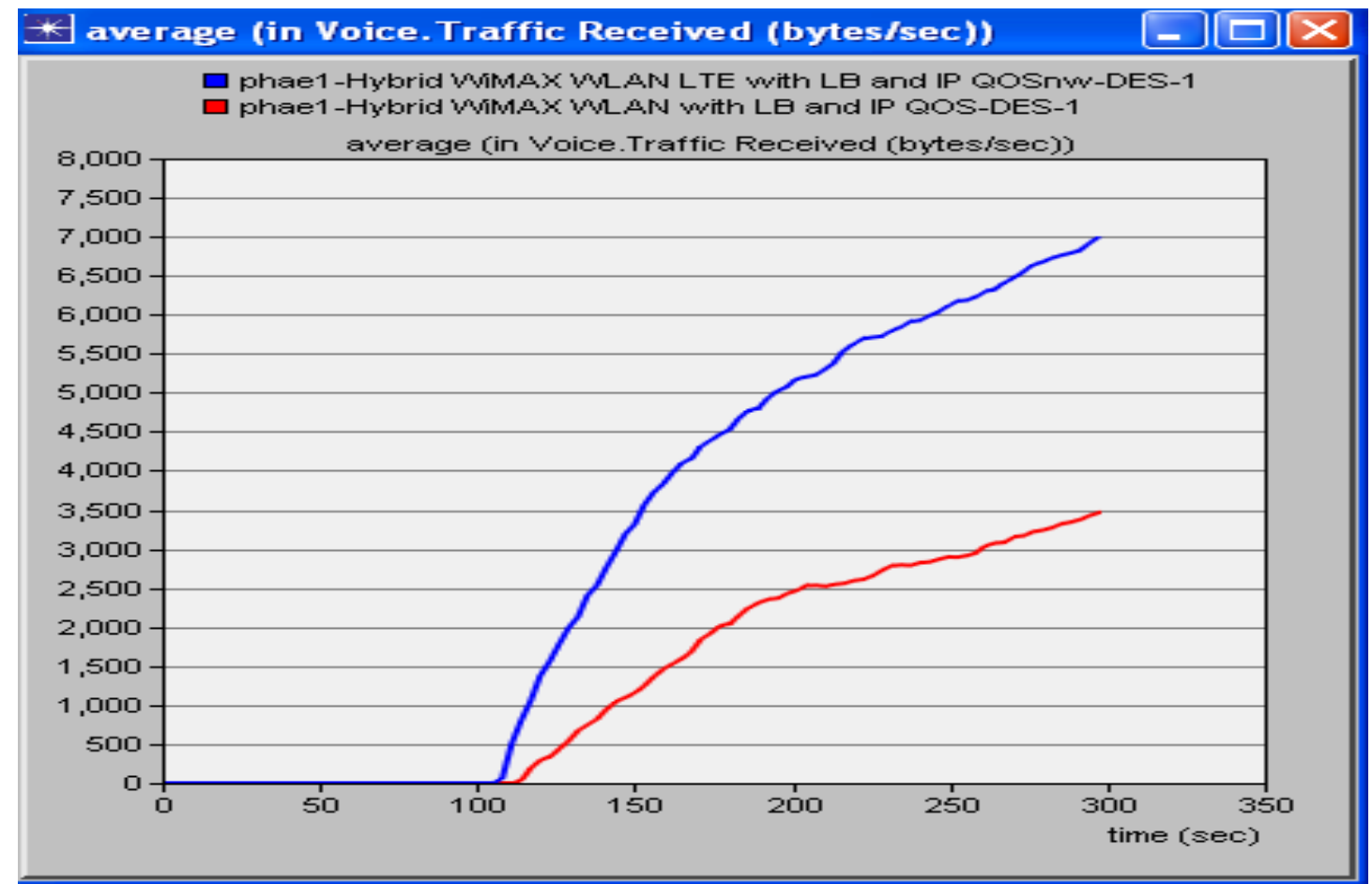

Figure 8: Voice Traffic Received (bytes/sec) for Hybrid coupled WiMAX-WLAN-LTE-LB-IPQOS and Hybrid coupled WiMAX-WLAN-LB-IPQOS

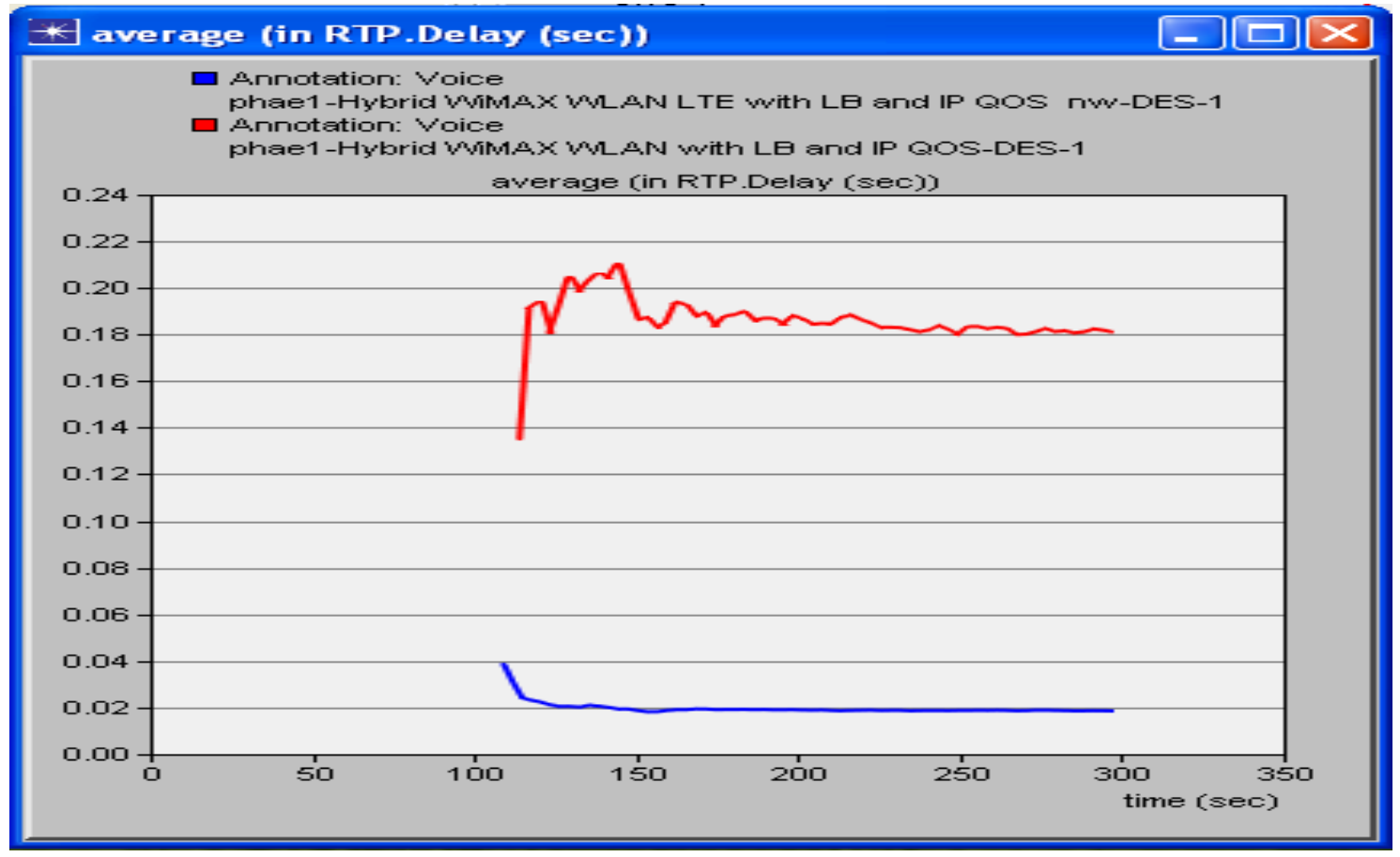

Figure 9: RTP delay (sec) for Hybrid coupled WiMAX-WLAN-LTE-LB-IPQOS and Hybrid coupled WiMAX-WLANLB-IPQOS

Voice Traffic Received (bytes/sec) is the average traffic arrival rate to all Voice applications deployed in the network. Figure 8 shows the Voice Traffic Received in hybrid coupled WiMAX-WLAN-LTE-LB-IPQoS and hybrid coupled WiMAX-WLAN-LB-IPQoS. It is observed that hybrid coupled WiMAX-WLAN-LTE-LB-IPQoS has more traffic sent compared to hybrid coupled WiMAX-WLAN-LBIPQoS. Figure 9 shows that RTP delay for hybrid coupled WiMAX-WLAN-LTE-LB-IPQoS and hybrid coupled WiMAX-WLAN-LB-IPQoS. It is observed that hybrid coupled WiMAX-WLAN-LTE-LB-IPQoS has delay compared to hybrid coupled WiMAX-WLAN-LB-IPQoS. 


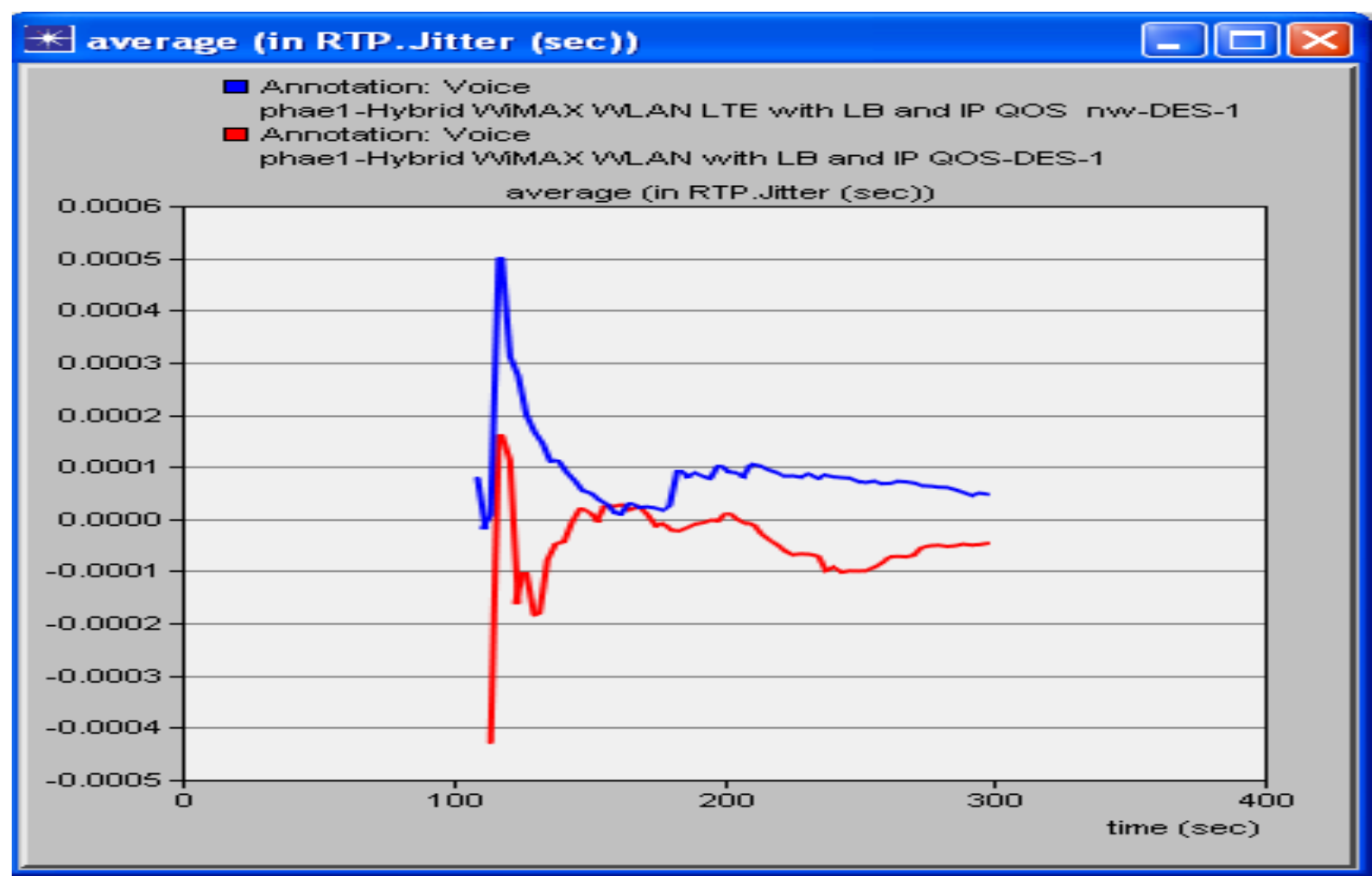

Figure 10: RTP Jitter (sec) for Hybrid coupled WiMAX-WLAN-LTE-LB-IPQOS and Hybrid coupled WiMAXWLAN-LB-IPQOS

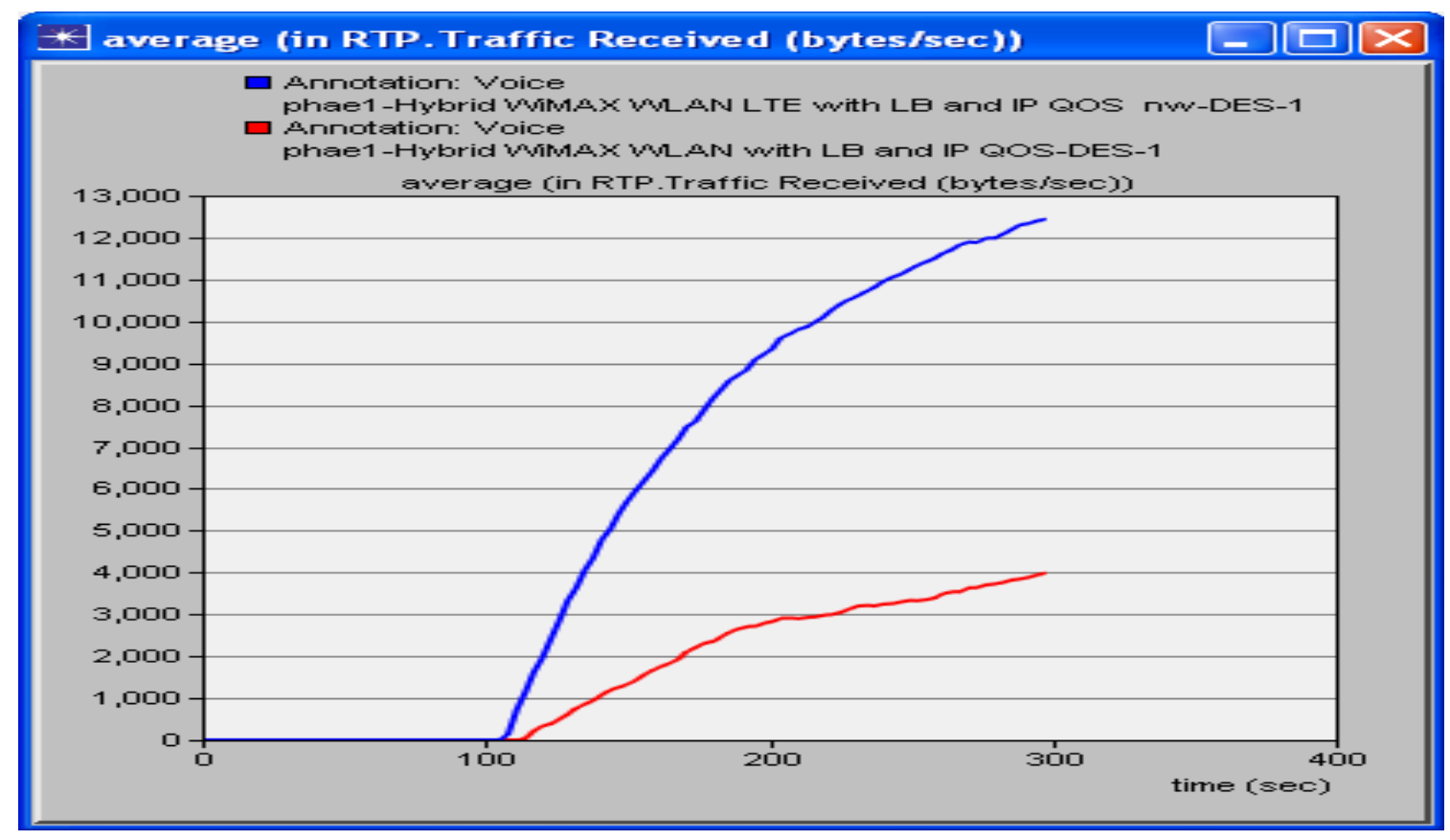

Figure 11: RTP Traffic Received (bytes/sec) for Hybrid coupled WiMAX-WLAN-LTE-LB-IPQOS and Hybrid coupled WiMAX-WLAN-LB-IPQOS

Figure 10 shows that RTP jitter for hybrid coupled WiMAXWLAN-LTE-LB-IPQoS and hybrid coupled WiMAXWLAN-LB-IPQoS. It is observed that hybrid coupled WiMAX-WLAN-LTE-LB-IPQoS has more jitter compared to hybrid coupled WiMAX-WLAN-LB-IPQoS. Figure 11 shows the RTP Traffic Received in hybrid coupled WiMAXWLAN-LTE-LB-IPQoS and hybrid coupled WiMAXWLAN-LB-IPQoS. It is observed that hybrid coupled WiMAX-WLAN-LTE-LB-IPQoS has more traffic sent compared to hybrid coupled WiMAX-WLAN-LB-IPQoS 
Table 1. Comparison table between Hybrid coupled WiMAX -WLAN-LB-IPQoS with Hybrid coupled WiMAX -WLANLTE- LB-IPQoS

\begin{tabular}{|l|l|l|}
\hline Parameter & $\begin{array}{l}\text { Hybrid coupled WiMAX \&WLAN with LB } \\
\text { \& IPQoS }\end{array}$ & $\begin{array}{l}\text { Hybrid coupled WiMAX -WLAN-LTE with LB } \\
\text { \& IPQoS }\end{array}$ \\
\hline $\begin{array}{l}\text { Voice : packet End to End } \\
\text { delay }\end{array}$ & $0.27 \mathrm{sec}$ & $0.23 \mathrm{sec}$ \\
\hline Voice : Traffic received & $3500 \mathrm{bytes} / \mathrm{sec}$ & $7000 \mathrm{bytes} / \mathrm{sec}$ \\
\hline Voice : Jitter & $0.00015 \mathrm{sec}$ & $0.00025 \mathrm{sec}$ \\
\hline Video : Traffic received & $600000 \mathrm{bytes} / \mathrm{sec}$ & $1500000 \mathrm{bytes} / \mathrm{sec}$ \\
\hline Video: End to End delay & $0.057 \mathrm{sec}$ & $0.075 \mathrm{sec}$ \\
\hline RTP : Traffic received & $4 \mathrm{Kbytes} / \mathrm{sec}$ & $12 \mathrm{Kbytes} / \mathrm{sec}$ \\
\hline RTP: Delay & $0.21 \mathrm{sec}$ & $0.04 \mathrm{sec}$ \\
\hline
\end{tabular}

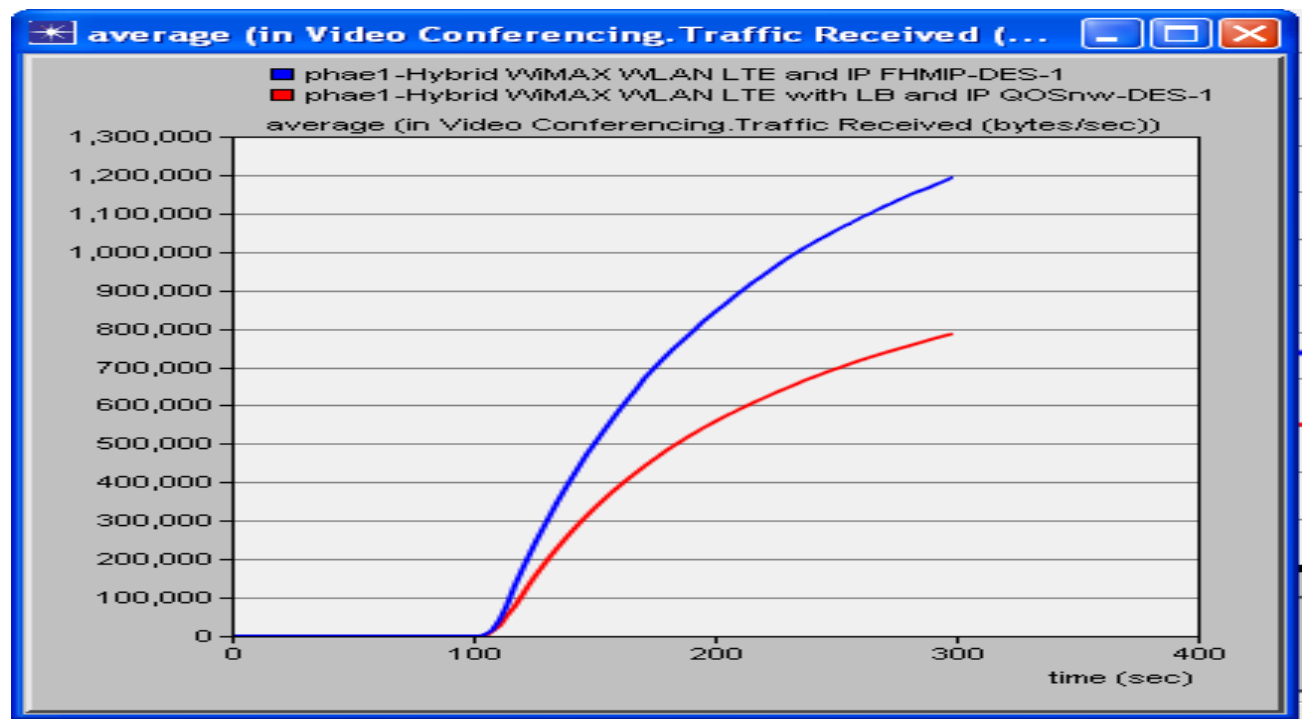

Figure 12: Video Conferencing Traffic Received (bytes/sec) for Hybrid coupled WiMAX-WLAN-LTE-LB-IPQOS and Hybrid coupled WiMAX-WLAN-LTE-LB-IPQOS using FHMIPv6

Video conferencing traffic received is the average traffic arrival rate to all Video Conferencing applications deployed in the network. Figure 12 shows that Video Conferencing Traffic Received in hybrid coupled WiMAX-WLAN-LTE-LB-IP
FHMIP and hybrid coupled WiMAX-WLAN-LTE-LBIPQoS. It is observed that hybrid coupled WiMAX-WLANLTE-LB-IPQoS carries more traffic compared to hybrid coupled WiMAX-WLAN-LB-IPQoS.

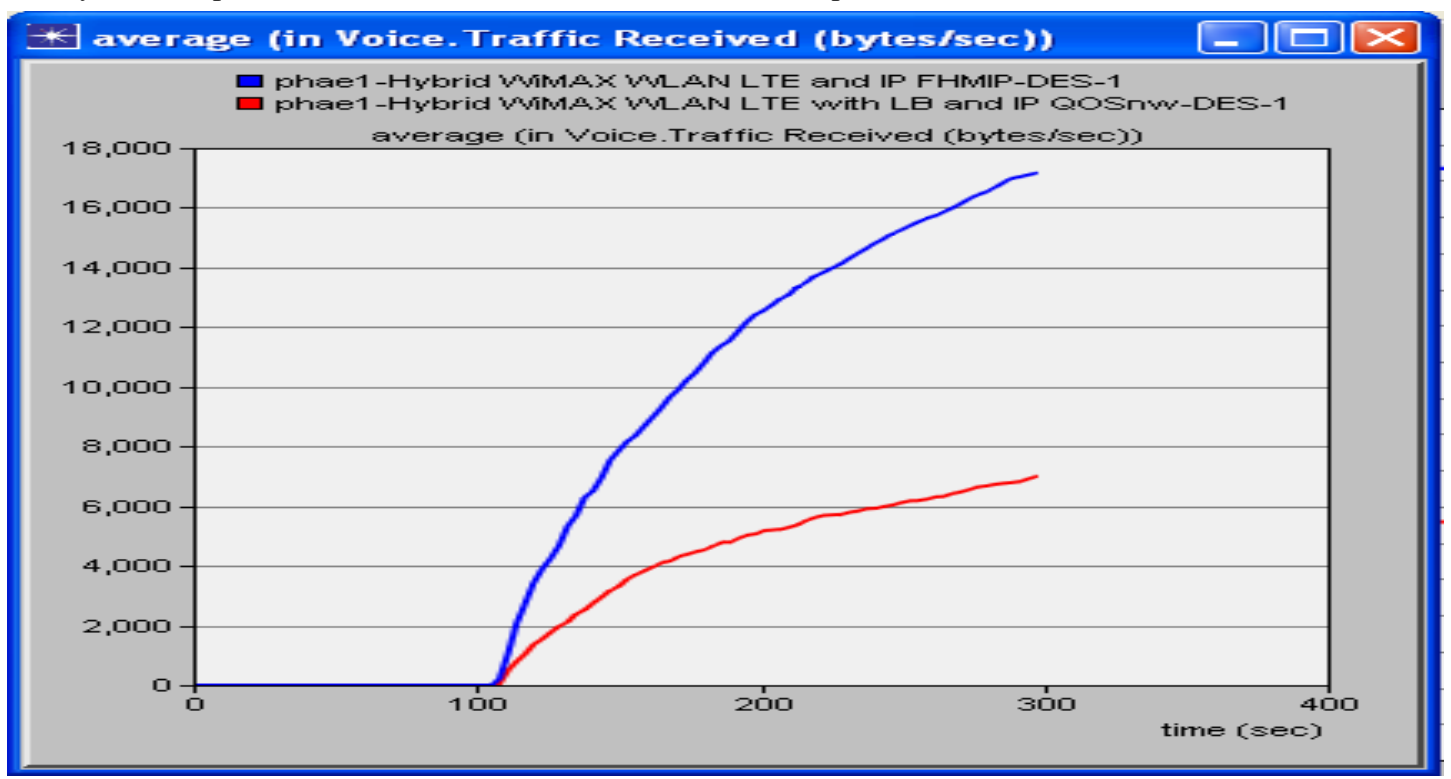

Figure 13: Voice Traffic Received (bytes/sec) for Hybrid coupled WiMAX-WLAN-LTE-LB-IPQOS and Hybrid coupled WiMAX-WLAN-LTE-LB-IPQOS using FHMIPv6 


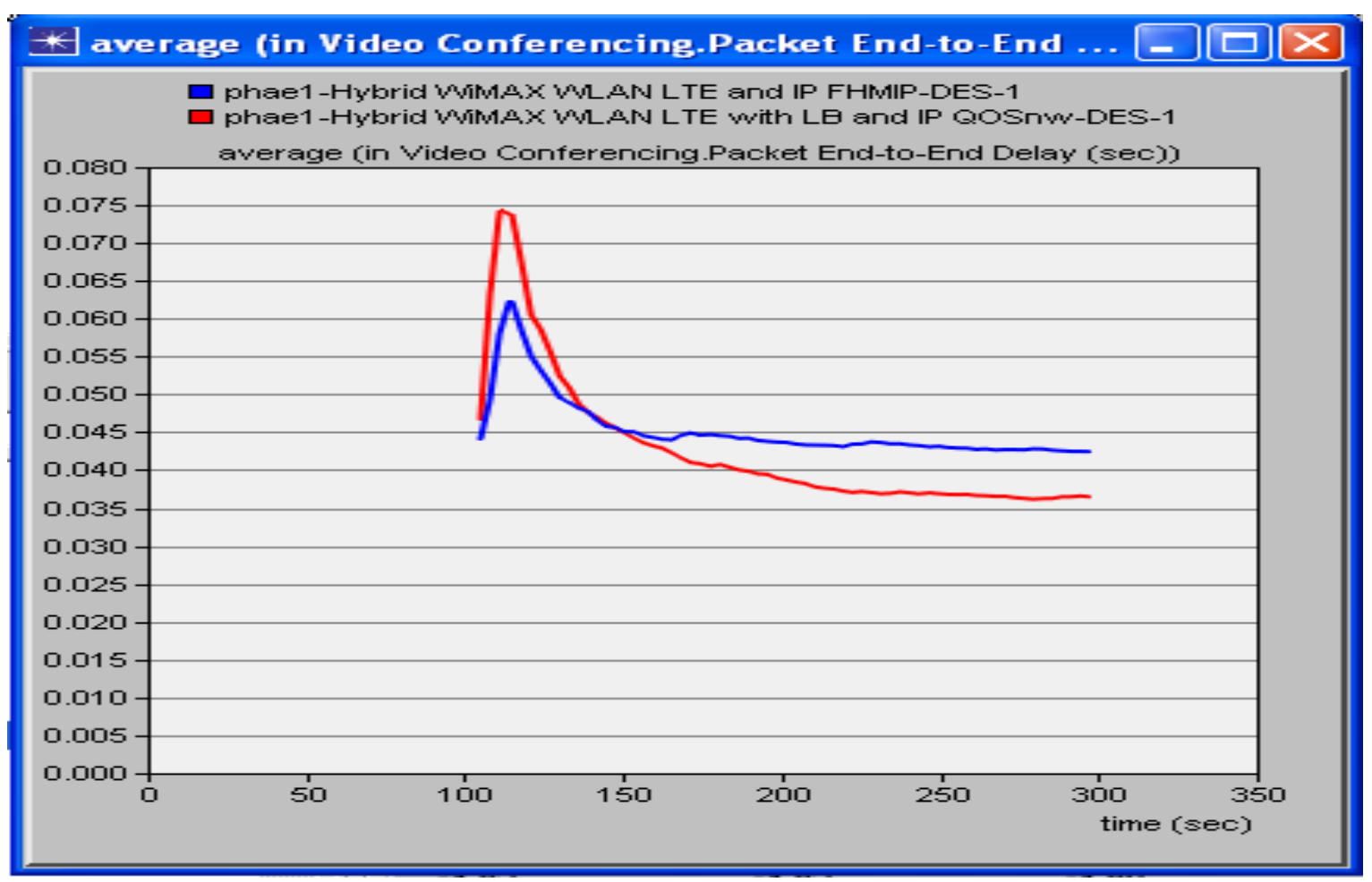

Figure 14: Video packet end to end delay for Hybrid coupled WiMAX-WLAN-LTE-LB-IPQOS and Hybrid coupled WiMAX-WLAN-LTE-LB-IPQOS using FHMIPv6

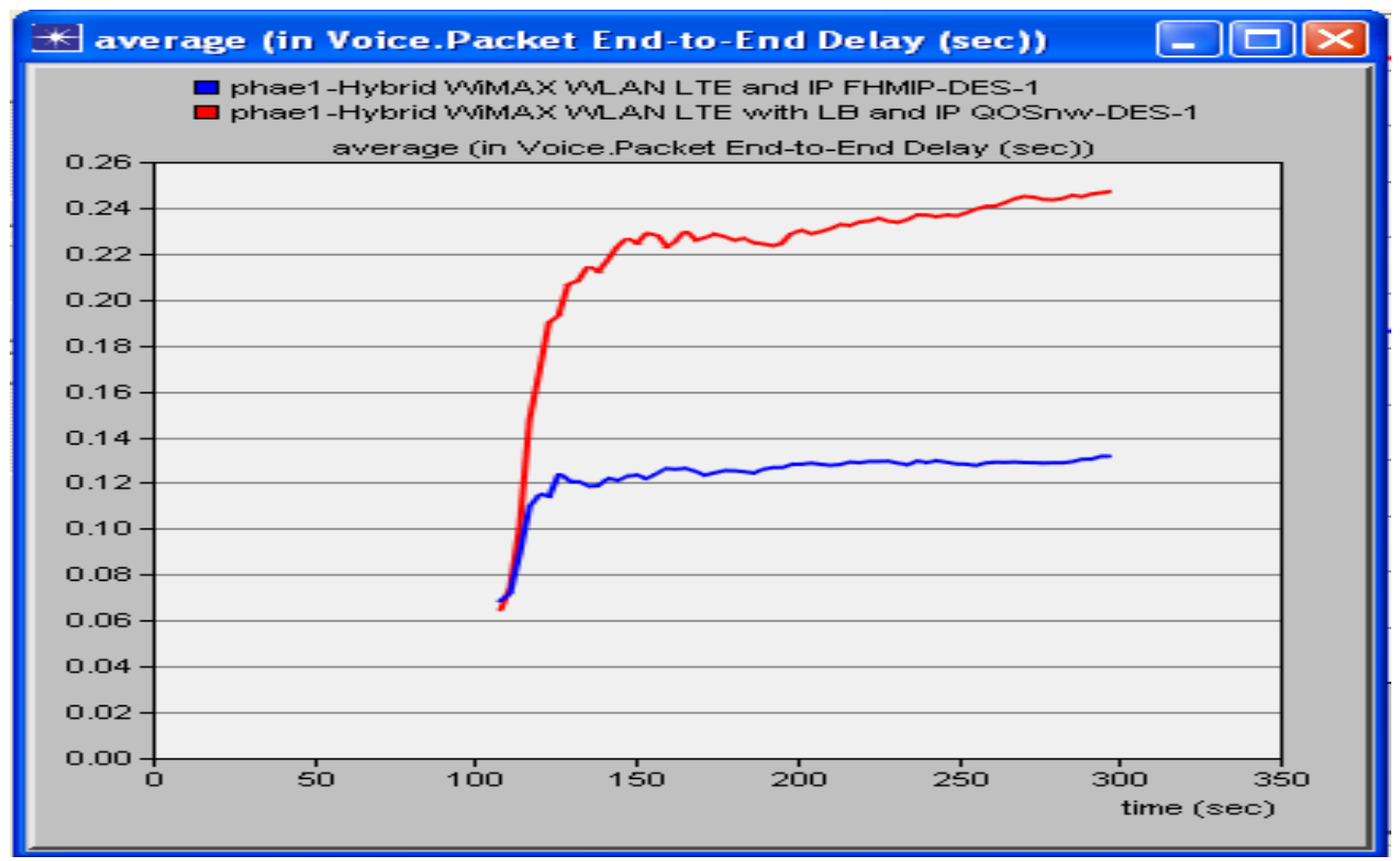

Figure 15: Voice packet end to end delay for Hybrid coupled WiMAX-WLAN-LTE-LB-IPQOS and Hybrid coupled WiMAX-WLAN-LTE-LB-IPQOS using FHMIPv6

Voice Traffic Received (bytes/sec) is the average traffic arrival rate to all Voice applications deployed in the network. Figure 13 shows the Voice Traffic Received in hybrid coupled WiMAX-WLAN-LTE-LB-IP FHMIP and hybrid coupled WiMAX-WLAN-LTE-LB-IPQoS. It is observed that hybrid coupled WiMAX-WLAN-LTE-LB-IPQoS carries more traffic compared to hybrid coupled WiMAX-WLAN-LB-IPQoS. Video Packet End-to-End Delay total is the delay experienced by the video packets. It includes network, encoding/decoding, and compression delays. Figure 14 shows the Video Packet End-to-End Delay in hybrid coupled WiMAX-WLAN-LTELB-IPQoS and hybrid coupled WiMAX-WLAN-LTE-LBIPQoS using FHMIPv6. It is observed that hybrid coupled WiMAX-WLAN-LTE-LB-IPQoS has greater delay of 7.5 millisec compared to hybrid coupled WiMAX-WLAN-LTELB-IP FHMIP which has 6.2milli sec. 
Voice Packet End-to-End Delay total is the delay experienced by the voice packets. It includes network, encoding/decoding, and compression delays. Figure 15 shows the Voice Packet End-to-End Delay in hybrid coupled WiMAX-WLAN-LTELB-IPQoS and hybrid coupled WiMAX-WLAN-LTE-LB-
IPQoS using FHMIPv6. It is observed that hybrid coupled WiMAX-WLAN-LTE-LB-IPQoS has greater delay of 0.23 sec compared to hybrid coupled WiMAX-WLAN-LTE-LB-IP FHMIP which has $0.12 \mathrm{sec}$.

Table 2. Comparison between Hybrid coupled WLAN-WiMAX-LTE-IPQoS-LB and Hybrid coupled WLAN-WiMAX-LTEIPQoS-LB-FHMIP.

\begin{tabular}{|l|l|l|}
\hline Parameter & $\begin{array}{l}\text { Hybrid coupled } \\
\text { WLAN-WiMAX- } \\
\text { LTE-IPQoS-LB }\end{array}$ & $\begin{array}{l}\text { Hybrid coupled } \\
\text { WLAN-WiMAX-LTE- } \\
\text { IPQoS-LB-FHMIP }\end{array}$ \\
\hline $\begin{array}{l}\text { Video: packet end to end delay } \\
\text { variation }\end{array}$ & $7.5 \mathrm{millisec}$ & $6.2 \mathrm{millisec}$ \\
\hline $\begin{array}{l}\text { Voice: packet end to end delay } \\
\text { variation }\end{array}$ & $0.23 \mathrm{sec}$ & $0.12 \mathrm{sec}$ \\
\hline Video:Traffic received & $800000 \mathrm{bytes} / \mathrm{sec}$ & $1200000 \mathrm{bytes} / \mathrm{sec}$ \\
\hline Voice: Traffic Received & $7000 \mathrm{bytes} / \mathrm{sec}$ & $17000 \mathrm{bytes} / \mathrm{sec}$ \\
\hline
\end{tabular}

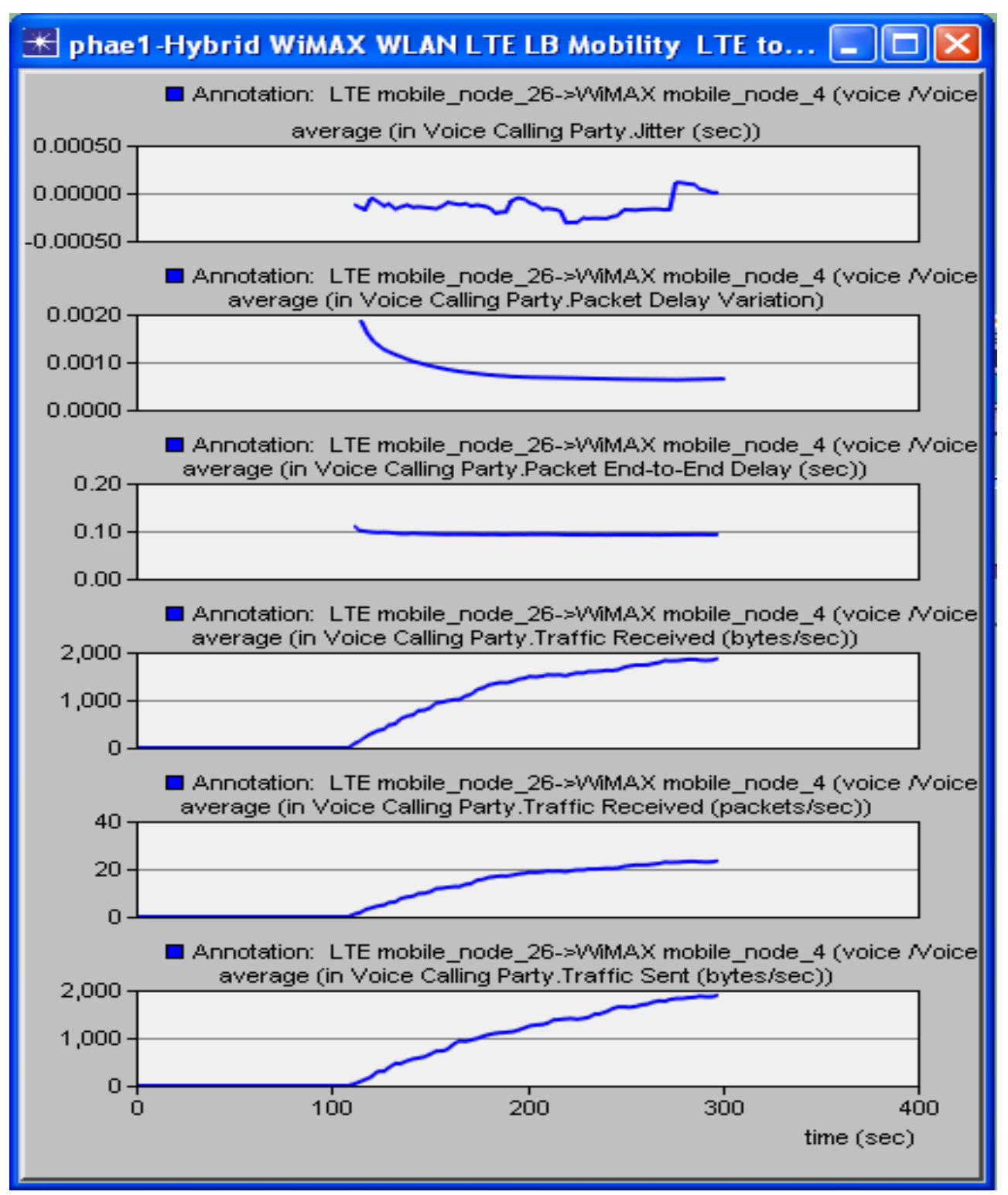

Figure 16: Voice Quality Parameters for Hybrid coupled WiMAX-WLAN-LTE-LB-IPQOS 
Figure 16 shows Voice Quality parameters such as jitter, packet delay variations, end-to-end delay, and traffic sent and received for the proposed Hybrid coupled WiMAX-WLANLTE-LB alone. In the proposed scenario mobility multimedia application configuration is made for all the users including all the technologies. Figure 5 to 15 discuss about Global user statistics and not the individual user statistics. Hence for example, LTE mobile node 26 communicating with WiMAX mobile node 4 is shown in the above figure for mobility. In this model, Voice communication alone shown for simple discussion. Here in the propose work Voice Jitter, end-to-end delay, Packet delay variation are very low for the individual users also. Traffic sent and received are also same for individual users. This shows proposed architecture outperforms well.

\section{CONCLUSION}

In this paper a hybrid coupled interworking model that integrates a WiMAX network, a WLAN network and a LTE network along with LB and IPQOS (WiMAX-WLAN-LTELB-IPQOS) is proposed and its performance is compared with hybrid coupled interworking of WiMAX and WLAN network with LB and IPQOS. The performance evaluation shows that the proposed architecture outperforms the existing technology. Hybrid coupled interworking of WiMAXWLAN-LTE-LB-IPQOS is also compared with hybrid coupled interworking of WiMAX-WLAN-LTE-LB-IPQOS using FHMIPV6 and it can be seen that the later outperforms well, since the advantage of both hierarchical mobile IPv6 and fast handover mobile IPv6 are combined together. In the near future incorporating Authentication, Authorization and Accounting (AAA) protocol into the proposed work of hybrid coupled WLAN-WiMAX-LTE-LB-IPQoS architecture will improve the network performance and decrease the handover delay.

\section{REFERENCES}

[1] Quoc-Thinh Nguyen-Vuong, Nazim Agoulmine, El Hadi Cherkaoui, and Laura Toni, "Multicriter ia Optimization of Access Selection to Improve the Quality of Experience in Heterogeneous Wireless Access Networks", IEEE Transactions On Vehicular Technology, vol. 62, no. 4, May 2013.

[2] Mian Guo, Shengming Jiang, Quansheng Guan and Huachoa Mao, "Provisioning of QoS Adaptability in Wired - Wireless Integrated Networks", IEEE Journal of Communication and networks, vol.15, February 2013.

[3] Perumalraja Rengaraju, Chung-Horng Lung, and F. Richard $\mathrm{Yu}$, Carleton University Anand Srinivasan, Eion Inc, "On QoE Monitoring and E2E Service Assurance in 4G Wireless Networks" IEEE Transactions Wireless Communications, August2012.

[4] C.Sarraf, F. Ousta. "End-to-End Quality of Services Issues in 4G Mobile Networks", 12th WSEAS International Conference on Communications, Crete Island, Greece, July 23-25 2008.

[5] D.G.Stratogiannis, et al., "4G Wireless Networks: Architectures, QoS Support and Dynamic Resource
Management",Wireless Network Traffic and Quality of Service Support: Trends and Standards, vol. 1, T. Lagkas, et al., ed. Information Science Reference, 2010.

[6] Y. Gwon, J. Kempf, and A. Yegin, "Scalability and robustness analysis of mobile IPv6, fast mobile IPv6, hierarchical mobile IPv6, and hybrid IPv6 mobility protocols using a large-scale simulation," in Proc. Of IEEE Conference on Communication (ICC'04), vol. 7, pp. 4087-4091, June 2004.

[7] Charles Sarrafl, FirasOusta, NidalKamel and MohdZuki, "Mapping of QoS between UMTS and WiMAX in Tight coupling Heterogeneous Wireless Network", International Journal of Soft Computing and Software Engineering, vol.2,no.3,2012.

[8] X.Fangmin, et al., "Interworking of Wimax and 3GPP networks based on IMS [IP Multimedia Systems (IMS) Infrastructure and Services]", Communications Magazine, IEEE, vol. 45, pp. 144-150, 2007.

[9] L.Zhang et al "Evaluating the Performance of Fast Handover for Hierarchical MIPv6 in Cellular Networks", Journal of Networks, vol. 3, no. 6, pp. 36-43, 2008.

[10] Wenxiao shi, Bin Li,NA Li and Chuanjun Xia, "A Network Achitecture for Load Balancing of Heterogeneous wireless Networks", IEEE journal of networks, vol.6,no.4,April 2011.

[11] G.Q. Ning, G.X.Zhu, "Load balancing based on traffic selection in heterogeneous overlapping cellular networks", Journal Of Computer Systems, vol.27, no.11, pp.2036-2041, Nov.2006.

[12] P.Andreas, et al " Force based laod balancing in colocated UMTS/GSM networks", IEEE Vehicular Technology, vol.6, no.6, pp.4402-4406,Srpt.2004.

[13] Ramon Ferrus, Oriol Sallent, and Ramon Agusti, Universitat Politecnica DeCatalunya,“ Interworking in Heterogeneous Wireless Networks: Comprehensive Framework and Future Trends", IEEE Wireless Communications, April 2010.

[14] A.Salkintzis, WLAN/3G Interworking Architectures for Next Generation Hybrid Data Networks", IEEE International Conference on Communications 2004, Vol.7, pp. 3984-3988.

[15] Ramon Ferrus, Oriol Sallent, and Ramon Agusti, Universitat Politecnica DeCatalunya, "Interworking in Heterogeneous Wireless Networks: Comprehensive Framework and Future Trends", IEEE Wireless Communications, April 2010.

[16] R.Beaubrun, "Integration of Heterogeneous Wireless Access Networks", Heterogeneous Wireless Access Networks, vol. 1, 1, ed. Springer, 2008.

[17] L. J. Zhang and S. Pierre, "Performance Analysis of Fast Handover for Hierarchical MIPv6 in Cellular Networks," in Proc. of IEEE 67th Vehicular Technology Conference (VTC2008-Spring), Marina Bay, Singapore, pp. 23742378, May 2008. 